\title{
Adherence of North-African Pulmonologists to the 2017-Global Initiative for Chronic Obstructive Lung Disease (GOLD) Pharmacological Treatment Guidelines (PTGs) of Stable Chronic Obstructive Pulmonary Disease (COPD)
}

\author{
Sana Aissa $\mathbb{D}^{1,2}$ Asma Knaz, ${ }^{1,2}$ Jihene Maatoug $\mathbb{D}^{3}{ }^{3}$ Ahmed Khedher, ${ }^{4}$ Wafa Benzarti, ${ }^{1,2}$ \\ Ahmed Abdelghani, ${ }^{1,2}$ Abdelhamid Garrouche, ${ }^{1,2}$ Abdelaziz Hayouni, ${ }^{1,2}$ \\ Mohamed Benzarti, 1,2 Imen Gargouri, 1,2 and Helmi Ben Saad iD 5,6,7 \\ ${ }^{1}$ Department of Pneumology, Farhat Hached University Hospital in Sousse, Tunisia \\ ${ }^{2}$ Interaction of the Cardiorespiratory System (LR14ES05) Research Laboratory, Faculty of Medicine of Sousse, \\ University of Sousse, Tunisia \\ ${ }^{3}$ Université de Sousse, Faculté de Médecine de Sousse, Département d'épidémiologie, Hôpital Farhat Hached, Sousse, Tunisia \\ ${ }^{4}$ Medical Intensive Care Unit, Farhat Hached University Hospital in Sousse, Tunisia \\ ${ }^{5}$ Physiology Laboratory, Faculty of Medicine of Sousse, University of Sousse, Tunisia \\ ${ }^{6}$ Department of Physiology and Functional Exploration, Farhat Hached University Hospital in Sousse, Tunisia \\ ${ }^{7}$ Heart Failure (LR12SP09) Research Laboratory, Farhat Hached University Hospital in Sousse, Tunisia
}

Correspondence should be addressed to Helmi Ben Saad; helmi.bensaad@rns.tn

Received 6 November 2019; Revised 13 February 2020; Accepted 17 February 2020; Published 28 February 2020

Academic Editor: Noriyoshi Sawabata

Copyright ( $) 2020$ Sana Aissa et al. This is an open access article distributed under the Creative Commons Attribution License, which permits unrestricted use, distribution, and reproduction in any medium, provided the original work is properly cited.

Background. No previous study has investigated the adherence rate of North-African pulmonologists to the 2017-GOLD PTGs. Aims. To investigate the adherence rate of Tunisian pulmonologists to the 2017-GOLD PTGs and to identify the barriers to their adherence. Methods. This was a cohort study involving clinically stable COPD patients who presented to a pulmonology outpatient consultation. The patients were classified as having been appropriately and inappropriately (over- or undertreatment) treated for the GOLD group. Logistic regression was performed to determine the adherence barriers to the 2017-GOLD PTGs. Results. A total of 296 patients were included (88.1\% males, mean age: $68 \pm 10$ years; GOLD A (7.1\%), B (36.1\%), C (4.1\%), and D (52.7\%)). The pulmonologists' adherence rate to the 2017-GOLD PTGs was $29.7 \%$. There was a significant statistical difference between the adherence rates among the four GOLD groups (A: $19.0 \%$, B: 20.6\%, C: $8.3 \%$, and D: $39.1 \% ; p=0.001$ ). Differences were statistically significant between the GOLD group D and groups B $(p=0.001)$ and C $(p=0.033)$. The multivariate analysis showed that age (odds ratio (OR): 0.968), socioeconomic level (high/medium vs. low; OR: 2.950), insurance type (national health insurance vs. others; OR: 2.851), and GOLD groups (A/B vs. C/D; OR: 3.009) significantly influenced the adherence rate to the 2017-GOLD PTGs. Conclusion. The adherence rate of Tunisian pulmonologists to the 2017-GOLD PTGs is low. It seems that the patients' age, socioeconomic level, national health insurance coverage, and GOLD groups influenced their adherence.

\section{Introduction}

The 2017-Global Initiative for Chronic Obstructive Lung Disease (GOLD) guideline, published online in December 2016, presented new recommendations regarding chronic obstructive pulmonary disease (COPD) pharmacologic and nonpharmacologic treatments [1]. It also provided suggestions with regard to the way pulmonologists can integrate these recommendations into COPD care practices $[1,2]$. However, although pulmonologists are the frontline healthcare 
professionals throughout the COPD continuum of care, their understanding of some GOLD pharmacological treatment guidelines (PTGs) is questioned [3,4]. First, a large inequality of pulmonologists' adherence rates to the GOLD PTGs was reported worldwide (e.g., rates varied from 26.3 (Greece [5]) to $61.4 \%$ (Italy [6]) in Europe, from 18.7\% [7] to 40\% [8] in the USA, and from $44.9 \%$ (Taiwan [9]) to $49.6 \%$ (South Korea [10]) in Asia, and a low adherence rate was reported in LatinAmerica (36.3\% in Brazil [11])). Second, several barriers were advanced to explain the aforementioned variability in the adherence rates to the GOLD PTGs $[1,12,13]$. According to the World Health Organization (WHO) [14], these barriers are frequently related to different aspects of the problem (e.g., social and economic factors, healthcare team/system, characteristics of the disease, disease therapies, and patient-related factors). In case of the COPD, the barriers can be related to the physician (e.g., knowledge, nonagreement, or nonfamiliarity with the recommendations, lack of awareness and/or of educational materi$\mathrm{al} /$ support, time constraints, and outcome of expectancy), patient (e.g., understanding, age, sex, race, smoking status, schooling level, socioeconomic level (SEL), length of COPD diagnosis, and number of concomitant treatments), guideline (e.g., presence and number of exacerbations, COPD assessment test (CAT) score, and disease severity), and/or social (e.g., treatment costs or availability, environmental factors) [5, 12-23].

In Africa, the data related to the pulmonologists' understanding of the 2017-GOLD PTGs (pulmonologists' adherence rates and/or barriers) are lacking. For example, in North-Africa (Tunisia, Algeria, Morocco, Libya, Mauritania, and Egypt), despite the fact that COPD is frequent [24], health authorities had no information regarding the pulmonologists' adherence rate and/or barriers to the 2017-GOLD PTGs [1]. To the best of the authors' knowledge, no previous North-African study has investigated the aforementioned issue. This crucial situation presents a handicap for any future planning action. In fact, pulmonologists' adherence to appropriate therapies is a major determinant of the treatment success. A low adherence reduces the optimum clinical benefits and therefore attenuates the overall efficiency of any health system [14].

According to the WHO [14], "additional research is needed on the rates of adherence and barriers to adherence in developing countries." Taking into account the abovementioned points, the main aim of this study was to determine the adherence rate of Tunisian pulmonologists to the 2017GOLD PTGs. The second aim was to identify their adherence barriers. Tunisian pulmonologists managing COPD are supposed to perfectly adhere to the 2017-GOLD PTGs.

\section{Population and Methods}

2.1. Study Design. This was a cohort study conducted from January to December 2017. It was carried out in the Pulmonology Department at Farhat Hached University Hospital (Sousse, Tunisia).

2.2. Population. The population source was formed by COPD patients presenting to the outpatient consultation of the abovementioned pulmonology department. The target popu- lation was formed by COPD patients who consulted during the study period. Only the patients with a confirmed COPD diagnosis and aged $\geq 40$ years at the moment of enrollment in the study were included. The patients with known concomitant chronic pulmonary diseases (e.g., asthma, lung cancer, and pulmonary fibrosis) or presenting an acute COPD exacerbation were not included.

2.3. Sample Size. The sample size was estimated using a predictive formula [25], detailed in Appendix A . The sample size for the study was 298 COPD patients.

2.4. Data Collection. COPD patients' data were collected from their sheets/records stored in the outpatient consultation archive. The pulmonology department, including ten pulmonologists (six professors, two assistants, and four residents), has an electronic database of all the examined patients. The patients' records are encoded using specific keywords. In this study, the applied keyword was "COPD." Each patient's sheet/record includes the following information: sociodemographic (i.e., sex, birth date, location of residence, SEL, and health insurance status), clinical (i.e., smoking habits, comorbidities), and COPD (i.e., spirometric data, dyspnea, GOLD stage/group, exacerbation history, and pharmacologic and nonpharmacologic treatments) characteristics. Incomplete coded sheets/records were excluded.

The following three sociodemographic data were noted: location of residence (urban/rural), SEL (low (unemployed), medium (manual employees), and high (skilled and professional employees)) [26], and health insurance status (indigent, national health insurance, and no insurance).

Cigarette smokers were divided into three groups [27]: nonsmokers, former smokers (smokers who had stopped smoking for more than one year), and current smokers (people who still smoke). Heavy smokers were patients who smoked more than 20 pack-years. Two groups of patients were identified according to whether they were exposed to biomass or not [28]. The patient's comorbidities were noted.

The following COPD characteristics were noted: (i) postbronchodilator (PBD) forced expiratory volume in one second $\left(\mathrm{FEV}_{1}, \mathrm{~L}, \%\right)$, forced vital capacity (FVC, L, \%), $\mathrm{FEV}_{1} / \mathrm{FVC}$ ratio (absolute value); (ii) GOLD stage; (iii) modified British Medical Research Council (mMRC) scale for dyspnea (detailed in Appendix A) [29]; (iv) exacerbation risk; and (v) GOLD group. Spirometric and bronchodilator tests were performed according to the international guidelines [30]. Local spirometric norms were used [31]. COPD exacerbation was defined as an acute event characterized by a deterioration of the patient's respiratory symptoms that are beyond the normal day-to-day variations and which leads to treatment modification [32].

The following pharmacologic treatments were noted: short-acting bronchodilators $\left(\beta_{2}\right.$-agonists (SABA), muscarinic agonists (SAMA)), long-acting bronchodilators $\left(\beta_{2}\right.$ agonists (LABA), muscarinic antagonists (LAMA)), inhaled corticosteroids (ICS), and theophylline. Only earlier longterm treatments used by stable COPD patients at the time of their inclusion in the study were considered. Long-term treatments were those prescribed by specialists at the time 
TABLE 1: Criteria of appropriate and inappropriate treatment according to the 2017-GOLD pharmacological treatment guidelines.

\begin{tabular}{|c|c|c|c|c|}
\hline \multirow{2}{*}{ GOLD group } & \multicolumn{2}{|c|}{ Appropriate treatment } & \multicolumn{2}{|c|}{ Inappropriate treatment } \\
\hline & First choice & Second choice & Undertreatment & Overtreatment \\
\hline A & Bronchodilator & Change the bronchodilator & No bronchodilator & $\begin{array}{c}\text { SABA-LAMA } \\
\text { SABA-ICS } \\
\text { SABA-ICS-Theo } \\
\text { SABA-ICS-LABA } \\
\text { SABA-ICS-LAMA } \\
\text { SABA-ICS-LAMA-LABA } \\
\text { SABA-ICS-LABA-Theo } \\
\text { SABA-LABA-LAMA }\end{array}$ \\
\hline B & LABA or LAMA & LAMA-LABA & $\begin{array}{l}\text { Only short-acting } \\
\text { bronchodilator }\end{array}$ & $\begin{array}{c}\text { SABA-ICS } \\
\text { SABA-ICS-LAMA } \\
\text { SABA-ICS-Theo } \\
\text { SABA-ICS-LABA } \\
\text { SABA-ICS-LABA-Theo } \\
\text { SABA-ICS-LABA-LAMA }\end{array}$ \\
\hline $\mathrm{C}$ & LAMA & $\begin{array}{c}\text { LABA-LAMA } \\
\text { (if persistence of exacerbations) } \\
\text { or LABA-ICS }\end{array}$ & $\begin{array}{c}\text { Only ICS } \\
\text { Only LABA } \\
\text { Only SABA } \\
\text { SABA-ICS } \\
\text { SABA-LABA } \\
\end{array}$ & $\begin{array}{c}\text { SABA-ICS-Theo } \\
\text { SABA-ICS-Theo-LABA }\end{array}$ \\
\hline $\mathrm{D}$ & LAMA-LABA & $\begin{array}{l}\text { LABA-ICS or LAMA-LABA-ICS } \\
\text { (if persistent symptoms } \\
\text { or acute exacerbations) }\end{array}$ & $\begin{array}{c}\text { Only ICS } \\
\text { Only LABA } \\
\text { Only ICS+LAMA } \\
\text { Only SABA } \\
\text { SABA-LABA } \\
\text { SABA-ICS } \\
\text { SABA-ICS-Theo } \\
\text { SABA-ICS-Theo-LABA } \\
\text { SABA-ICS-Theo-LAMA }\end{array}$ & \\
\hline
\end{tabular}

GOLD: Global Initiative for Chronic Obstructive Lung Disease; ICS: inhaled corticosteroids; LABA: long-acting $\beta$-agonist; LAMA: long-acting muscarinic antagonist; SABA: short-acting bronchodilator agonist; Theo: theophylline.

of inclusion in the database and which were extended several months later. Nonpharmacologic treatments included smoking cessation, influenza vaccination, pulmonary rehabilitation, long-term oxygen, and noninvasive ventilation.

2.5. Applied Definitions and Classifications. COPD diagnosis was retained when the $\mathrm{PBD} \mathrm{FEV}_{1} / \mathrm{FVC}$ ratio $<0.70$ [1]. Four GOLD stages (mild, moderate, severe, and very severe), detailed in Appendix A, were identified [1]. The refined "ABCD" assessment tool derived exclusively from the patient's symptoms (dyspnea, mMRC) and the exacerbation history (including prior hospitalizations) was applied [1]. Four GOLD groups (A, B, C, and D) were categorized [1]. The patients who developed two or more exacerbations per year were qualified as frequent exacerbators [33].

Pulmonologists' adherence to the guidelines was defined as "conformity in fulfilling or following official, recognized, or institutional requirements, guidelines, recommendations, protocols, pathways, or other standards" [34]. The appropriateness of the pharmacologic treatment and the type of inappropriateness were established in accordance with the 2017GOLD PTGs [1]. Appropriate treatment was defined as using the first drug choice or the alternative choice recommended in the 2017-GOLD PTGs [1]. Inappropriate therapy was classified as over- or undertreatment in accordance with the cat- egorization in the GOLD guidelines. In clinically stable COPD patients, the 2017-GOLD PTGs are dependent on the GOLD groups (Table 1) [1].

2.6. Statistical Analysis. The Kolmogorov-Smirnov test was used to analyze the distribution of variables. When the distribution was normal and the variances were equal, the results were expressed by their mean \pm standard deviation and $95 \%$ confidence interval. When the distribution was not normal, the results were expressed by their median (interquartile range). Qualitative data were expressed as relative number (\%). The categorical variables were analyzed using a chisquare test for independent samples. Comparisons of the percentages of patients adhering to the 2017-GOLD PTGs across the GOLD groups were accomplished via the Pearson chi-square test. When applicable, significant differences between the percentages were tested using the McNemar test. The factorial analysis of variance was used to compare the quantitative variables. It was then followed by the Tukey test. The odds ratio (OR) was calculated using the logistic regression test during the uni- and multivariate analysis. Only identified significant factors during the univariate analysis were included in the multivariable one. The level of statistical significance was set at 0.05 . All statistical analyses were performed using SPSS 20.0 software. 


\section{Results}

3.1. General Characteristics. Among the 350 medical records, 54 were excluded mainly because of missing data. Table 2 displays the general characteristics of the 296 COPD patients.

3.2. Tunisian Pulmonologists' Adherence Rates to the 2017GOLD PTGs. There was a significant difference between the pulmonologists' adherence rates to the 2017-GOLD PTGs across the GOLD groups (Figure 1). The difference was significant between the group $\mathrm{D}(39.1 \%)$ and the groups $\mathrm{B}$ (20.6) and C (8.3\%). The overall adherence rate was $29.7 \%$ $(n=88)$.

For each GOLD group, there was a significant difference between the percentages of patients with appropriate and inappropriate pharmacologic treatments (Table 3 ). The most inappropriate pharmacologic treatments were the associations SABA-ICS in groups A (33.3\%) and B (29.9\%), SABA-LABA-ICS-Theophylline in group C (33.3\%), and SABA-ICS-Theophylline $(21.1 \%)$ as well as SABA-ICS (19.9\%) in group D.

3.3. Adherence Barriers to the 2017-GOLD PTGs. Twelve factors influenced the Tunisian pulmonologists' adherence to the 2017-GOLD PTGs (Table 4): sex, age (absolute data and younger group), SEL, national health insurance coverage, smoking habits (current or ex-smoker and exposition to biomass smoke), follow-up by a pulmonologist, frequent exacerbator, and GOLD groups. The multivariate analysis retained only the following four factors: age, SEL, national health insurance coverage, and GOLD groups (Table 5).

\section{Discussion}

The main finding of this study involving 296 COPD patients was a low adherence rate $(29.3 \%)$ of Tunisian pulmonologists to the 2017-GOLD PTGs. It seems that the patients' age, SEL, national health insurance coverage, and GOLD groups influenced the Tunisian pulmonologists' adherence to the 2017GOLD PTGs.

In developing countries, such as the North-African ones, when combined with a meagre access to healthcare, a lack of correct diagnosis, and a restricted access to medicines, low adherence represents a challenge to treat chronic conditions, such as COPD [14]. To the best of the authors' knowledge, this study is the first North-African and Maghrebi one raising the issue of pulmonologists' adherence to the 2017-GOLD PTGs. Table 1S (Appendix A) summarizes the adherence variability to some GOLD PTGs observed in the literature. The rational of the study is highlighted in Appendix A.

4.1. Discussion of Methodology. Discussion related to the sample size and the inclusion criteria is detailed in Appendix A. This study presented some limitations. The first one concerns the adherence concept itself. Although this concept looks clear, its operationalization and its evaluation methods vary among studies and clinical settings [35]. Moreover, the discussion of different but related concepts, including adherence, compliance, concordance, persistence, and some associated terms (e.g., poor, suboptimal, and low), may increase confusion [36]. The aforementioned point was perfectly discussed by López-Campos et al. [12]. In the same line, the factors for analysis of pulmonologists' adherence were collected from the patient factors only, and it was better to collect the pulmonologist factors (e.g., by interviewing them) into data analysis. The second limitation is related to the use of patients' medical records for medical research [37]. In fact, these records are intended for healthcare purposes and not for research. They are therefore not structured to ease the research process [37]. Hence, the data quality of the patients' medical records is a serious obstacle to be taken into account during the study, as poor quality data can lead to biased results [37]. Since incomplete coding of all the patients' data might increase bias in case ascertainments [37], 54 records were excluded from this study. However, the use of medical records for medical research had some advantages such as access to data that are not easily available, large size of data sets, correct identification of COPD, and correct documentation of prescribed therapies [37]. Additionally, the design of the database is set before carrying out the study. So, the study is a post hoc analysis of previously recorded data. The third limitation concerns the lack of patients' record anonymity and the failure to obtain informed consent $[37,38]$. In similar studies, obtaining informed consent is expensive and timeconsuming. It also leads to bias in terms of responders and nonresponders, and it decreases the generalizability of the research results [38]. Given the lack of patients' contact or intervention, the authors of this study, and after consulting the hospital institutional review board, judged that there was no need for an ethics committee approval. The fourth limitation is related to the study design itself [39]. On the one hand, cohort studies may suffer from selection bias in addition to possible confounding by indication [39]. On the other hand, cohort studies form an appropriate study design to assess associations between multiple exposures and outcomes [39]. They are particularly applicable to study exposures for which randomization is not conceivable for practical or ethical reasons [39]. The last limitation is related to the study one-center design. It was better to include at least one additional center. The benefits of multicenter trials are numerous: rapid recruitment of sufficient numbers of patients and clearer results that are more convincing and more accepting, because the patient sample of multicenter studies is supposed to be representative. However, while Sousse had two university hospitals, only one pulmonology department exists.

4.2. Discussion of Results. The evaluation of pulmonologists' adherence to the 2017-GOLD PTGs represents a crucial element in the therapeutic approach for patients with COPD and a current test in hospitals [12]. An effective management requires pulmonologists to correctly adhere to the relevant clinical practice guidelines. This study is the first real-life study in Tunisia and the Maghreb reflecting the clinical practice of pulmonologists through an observational analysis of their adherence to the 2017-GOLD PTGs.

4.2.1. Pulmonologists' Adherence Rates to the 2017-GOLD PTGs. The overall adherence rate of Tunisian pulmonologists 
TABLE 2: General characteristics of the chronic obstructive pulmonary disease (COPD) patients $(n=296)$.

\begin{tabular}{|c|c|c|}
\hline \multirow{4}{*}{ Sociodemographic data } & Male sex & $261(88.1)$ \\
\hline & Age (years) & $68 \pm 10[66$ to 69$](41-89)$ \\
\hline & Younger group & $123(41.5)$ \\
\hline & Urban origin & $220(74.3)$ \\
\hline Socioeconomic level & High or medium & $236(79.7)$ \\
\hline National health insurance coverage & Yes & $201(67.9)$ \\
\hline \multirow{9}{*}{ Comorbidity type } & Arterial hypertension & $62(20.9)$ \\
\hline & Diabetes mellitus & $51(17.2)$ \\
\hline & Coronaropathy & $37(12.5)$ \\
\hline & Heart rhythm disorder & $32(10.8)$ \\
\hline & Heart failure & $21(7.0)$ \\
\hline & Dyslipidemia & $13(4.3)$ \\
\hline & Arterial occlusion of the lower limbs & $8(2.7)$ \\
\hline & Brain stroke & $6(2.0)$ \\
\hline & Dysthyroidism & $3(1.0)$ \\
\hline \multirow[t]{2}{*}{ Comorbidity } & Yes & $114(38.5)$ \\
\hline & Current or ex-smoker & $264(89.1)$ \\
\hline \multirow{3}{*}{ Smoking habits } & Biomass smoke & $35(11.8)$ \\
\hline & Mean smoking (pack-years) & $61 \pm 31[57$ to 65$](5-190)$ \\
\hline & Heavy smokers & $251(84.7)$ \\
\hline \multicolumn{3}{|l|}{ COPD history } \\
\hline \multirow{2}{*}{ COPD duration } & Years & $5 \pm 5[4$ to 6$](0-40)$ \\
\hline & Duration $<5$ years & $188(63.5)$ \\
\hline \multirow{3}{*}{ Follow-up before the inclusion in the study } & Pulmonologist & $193(65.2)$ \\
\hline & No follow-up & $60(20.2)$ \\
\hline & General practitioner & $43(14.5)$ \\
\hline \multirow{2}{*}{ Mean annual number of exacerbation } & Number & $3 \pm 1[2$ to 3$](0-10)$ \\
\hline & Frequent exacerbators & $154(52.1)$ \\
\hline \multirow{2}{*}{ Mean number of hospitalizations for acute exacerbation } & All live & $1.9 \pm 1.9[1.7$ to 2.2$](0.0-15.0)$ \\
\hline & 1 year before the inclusion & $0.9 \pm 1.0[0.8$ to 1.0$](0.0-13.0)$ \\
\hline \multicolumn{3}{|l|}{ Functional characteristics and GOLD groups } \\
\hline Postbronchodilator $\mathrm{FEV}_{1}$ & $(\%)$ & $46 \pm 21[44$ to 48$](18-118)$ \\
\hline \multirow{4}{*}{ Airflow limitation: GOLD } & 1 & $27(9.1)$ \\
\hline & 2 & $71(23.9)$ \\
\hline & 3 & $118(39.8)$ \\
\hline & 4 & $81(27.3)$ \\
\hline \multirow{4}{*}{ GOLD groups } & A & $21(7.1)$ \\
\hline & $\mathrm{B}$ & $107(36.1)$ \\
\hline & $\mathrm{C}$ & $12(4.1)$ \\
\hline & $\mathrm{D}$ & $156(52.7)$ \\
\hline \multicolumn{3}{|l|}{ Treatments } \\
\hline \multirow{8}{*}{ Pharmacological } & SABA-ICS & $71(23.9)$ \\
\hline & SABA-ICS-Theo & $49(16.5)$ \\
\hline & SABA-ICS-Theo-LABA & $43(14.5)$ \\
\hline & SABA-LABA-ICS & $31(10.4)$ \\
\hline & SABA-LABA-ICS-LAMA & $29(9.7)$ \\
\hline & SABA-LABA-LAMA & $28(9.4)$ \\
\hline & SABA & $24(8.1)$ \\
\hline & SABA-LABA & $15(5.1)$ \\
\hline
\end{tabular}


TABLE 2: Continued.

\begin{tabular}{lcc}
\hline & $5(1.6)$ & $1(0.3)$ \\
& SABA-LAMA & $251(84.7)$ \\
Observance of the pharmacological treatments & SABA-LAMA-ICS-Theo & $147(55.6)$ \\
& Yes & $45(15.2)$ \\
Nonpharmacological & Smoking cessation among the 264 smokers & $66(22.2)$ \\
& Noninvasive ventilation & $57(19.2)$ \\
\hline
\end{tabular}

Quantitative data were expressed as mean $\pm S D$ [95\% confidence interval] (min-max). Qualitative data were expressed as number (\%). FEV $_{1}$ : forced expiratory volume in one second; ICS: inhaled corticosteroid; LABA: long-acting $\beta_{2}$-agonist; LAMA: long-acting muscarinic antagonist; SABA: short-acting $\beta_{2}$-agonist; Theo: theophylline.

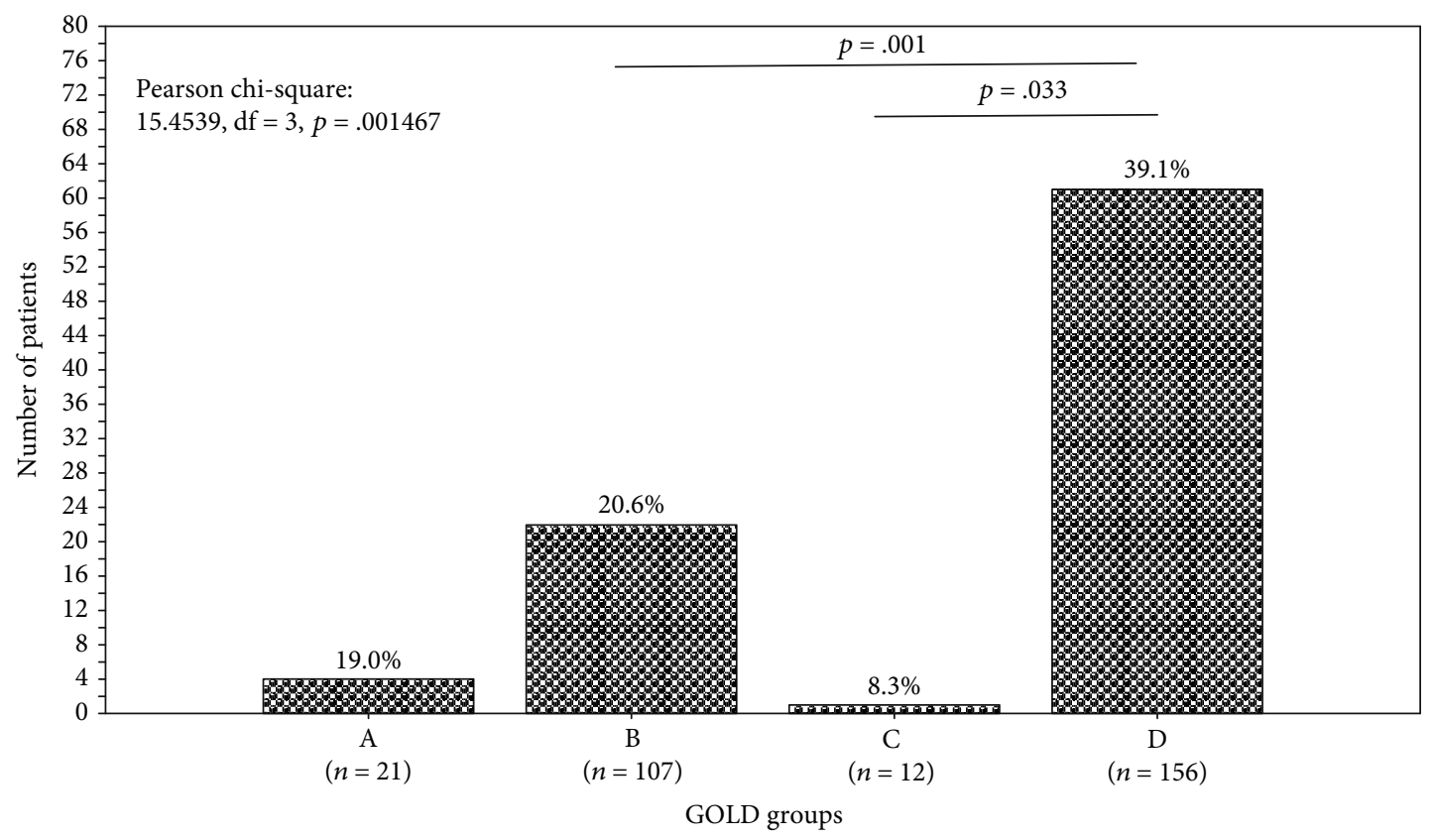

FIGURE 1: Number (\%) of patients having an appropriate medical treatment according to the 2017-GOLD guidelines across the four ABCD GOLD groups. GOLD: Global Initiative for Chronic Obstructive Lung Disease.

to the 2017-GOLD PTGs was 29.7\%. This low adherence rate was similar to that observed in a study in Greece [5] (26.3\%), but it was lower than others observed in Taiwan [9] (44.9\%) and in South Korea [10] (49.6\%) (Table 1S). A recent review including 11 studies performed in multiple countries across the globe showed a significant variability in the adherence rates to the GOLD PTGs [40]. For example, the pulmonologists' adherence rates to some other GOLD PTGs varied from 70.1\% (Greece) [41] to $18.7 \%$ (USA) [7] (Table 1S). The authors of an Italian study including 12 general practitioners reviewed 437 charts and revealed that only $38 \%$ of prescriptions were appropriate [42]. In this study, the pulmonologists' adherence rate was dependent on the COPD ABCD groups. The adherence rates were 19.0, $20.6,8.3$, and $39.1 \%$, in groups $\mathrm{A}, \mathrm{B}, \mathrm{C}$, and $\mathrm{D}$, respectively (Figure 1). However, the above rates were almost lower than the ones reported by Palmiotti et al. [13] (51.0, 54.2, 58.5, and $86.0 \%$, in groups $\mathrm{A}, \mathrm{B}, \mathrm{C}$, and $\mathrm{D}$, respectively). According to
Palmiotti et al. [13], the tendency in Italy is to prescribe the maximum therapy to all patients, irrespective of the degree of severity. Yet, the Tunisian pulmonologists' adherence rates were intermediate compared to those reported in similar studies (Table 1S). The adherence rates varied from 0.2 [43] to $61.5 \%$ [16] in group A, from 0.2 [43] to $43.9 \%$ [9] in group $\mathrm{B}$, from 0.0 [43] to $81.5 \%$ [10] in group C, and from 4.6 [10] to $96.2 \%$ [41] in group D. To summarize, COPD patients continue to be treated in the wrong way, signifying that the GOLD PTGs are not being completely utilized [13]. This frequent pattern is not specific to the developing countries, but it is rather an international issue (Table 1S).

In this study, ICS was prescribed in $75.7 \%$ of the patients (Table 2). The problem of overprescription of ICS was highlighted in the literature [5, 13, 44, 45]. For example, ICS (alone or with other medicines) was prescribed in 89, 85, 68.4, and 50\% of Turkish [44], Greek [45], Greek [5], and Italian [13] COPD patients, respectively. Ten reasons 


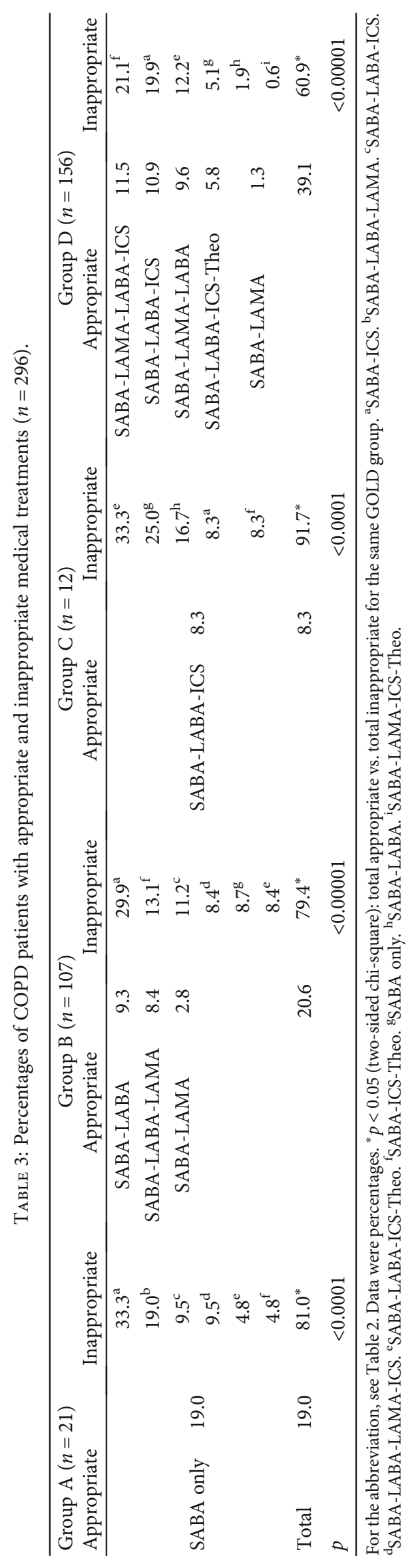


TABLE 4: Simple univariate analysis: influencing factors of the Tunisian pulmonologists' adherence to the 2017-GOLD pharmacological treatment guidelines $(n=296)$.

\begin{tabular}{|c|c|c|c|}
\hline Factors & Description & Odds ratio (95\% confidence interval) & $p$ \\
\hline \multirow{4}{*}{ Sociodemographic data } & Sex (female) & 2.797 (1.048 to7.469) & 0.0400 \\
\hline & Age (years) & $0.971(0.947$ to 0.997$)$ & 0.028 \\
\hline & Younger group & $1.744(1.054$ to 2.885$)$ & 0.0303 \\
\hline & Urban origin & $1.146(0.642$ to 2.046$)$ & 0.6427 \\
\hline Socioeconomic level & High or medium & $4.792(1.977$ to 11.611$)$ & 0.0005 \\
\hline National health insurance coverage & Yes & $4.205(2.153$ to 8.210$)$ & $<0.0001$ \\
\hline Comorbidity & Yes & $1.509(0.909$ to 2.504$)$ & 0.1114 \\
\hline \multirow{3}{*}{ Smoking habits } & Current or ex-smoker & $3.266(1.110$ to 9.611$)$ & 0.0316 \\
\hline & Biomass smoke & $0.357(0.133$ to 0.954$)$ & 0.0400 \\
\hline & Heavy smokers & $1.578(0.744$ to 3.347$)$ & 0.2344 \\
\hline COPD mean duration & $<5$ years & $0.714(0.428$ to 1.191$)$ & 0.1971 \\
\hline Follow-up before the inclusion in the study & Pulmonologist (vs. others) & $2.460(1.379$ to 4.386$)$ & 0.0023 \\
\hline Frequent exacerbator & Yes & $0.495(0.297$ to 0.826$)$ & 0.007 \\
\hline Postbronchodilator $\mathrm{FEV}_{1}(\%)$ & & $0.997(0.985$ to 1.009$)$ & 0.611 \\
\hline GOLD stages & $1-2$ (vs. 3-4) & $0.990(0.582$ to 1.682$)$ & 0.9709 \\
\hline GOLD groups & A-B (vs. C-D) & $0.435(0.255$ to 0.742$)$ & 0.0022 \\
\hline \multirow{4}{*}{ Nonpharmacological treatment } & Smoking cessation among the 269 smokers & $1.683(0.987$ to 2.870$)$ & 0.0556 \\
\hline & Noninvasive ventilation & $1.723(0.893$ to 3.325$)$ & 0.1043 \\
\hline & Long-term oxygen & $1.616(0.909$ to 2.874$)$ & 0.1021 \\
\hline & Influenza vaccination & $1.810(0.992$ to 3.302$)$ & 0.0528 \\
\hline
\end{tabular}

For abbreviations, see Table 2. $p$ (probability): logistic regression.

TABLE 5: Multivariate analysis: influencing factors of the Tunisian pulmonologists' adherence to the 2017-GOLD pharmacological treatment guidelines $(n=296)$.

\begin{tabular}{lccc}
\hline Factors & Description & Adjusted odds ratio (95\% confidence interval) & $p$ \\
\hline Age & Years & $0.968(0.941$ to 0.996$)$ & 0.025 \\
Socioeconomic level & High or medium & $2.950(1.205$ to 7.223$)$ & 0.018 \\
National health insurance coverage & Yes & $2.851(1.421$ to 5.720$)$ & 0.003 \\
GOLD groups & A-B (vs. C-D) & $3.009(1.691$ to 5.355) & $<0.0001$ \\
\hline
\end{tabular}

For abbreviations, see Table 2. $p$ (probability): logistic regression.

can be advanced to explain this ICS over prescription: (i) ICS is a treatment always available in hospitals; (ii) pulmonologists aim to cover all the stages/groups of COPD; (iii) lack of awareness with regard to the 2017-GOLD PTGs, even among pulmonologists [46]; (iv) repetition of the drugs previously prescribed by another physician without a cautious assessment [13]; (v) presence of wheezing [47]; (vi) high CAT score [47]; (vii) a belief that ICS are more effective [13]; (viii) higher preference for combination devices, including ICS-LABA rather than ICS and LABA in separate devices [13]; and (ix) information received from pharmaceutical companies [13]. In this study, the most inappropriate medical treatments were the associations SABA-ICS in groups A (33.3\%) and B (29.9\%), SABA-LABA-ICS-Theophylline in group C (33.3\%), and SABA-ICS-Theophylline $(21.1 \%)$ or SABA-ICS (19.9\%) in group D (Table 3). Other related studies reported very large frequencies of overprescription (from $85.4 \%$ [10] to $2.4 \%$ [41]). In this study, prescription of the inappropriate association SABA-ICS is justified by the fact that these two medicines are the only ones available in Tunisian hospitals. In a study in Greece [41], overprescription was identified in all GOLD groups. In COPD GOLD group D, the LABA-ICS-LAMA triple therapy was consistently the most prescribed treatment in many studies $[16,48]$. A Taiwanese study explained ICS overprescription in patients belonging to GOLD groups A/B and group D by the presence of wheezing and a high CAT, respectively [47].

The practical management of a complex disease, such as COPD, takes into account the interplay of the clinical severity, the patient's overall well-being, the physicians' experience, and the systems' based resources [49]. Four reasons could be advanced to explain the low adherence rate of Tunisian pulmonologists to the 2017-COPD PTGs: (i) the nonavailability of several treatments in Tunisian public hospitals. In fact, Tunisian hospitals provide SABA, ICS, and some oral bronchodilators. Patients have no access 
to LABA. (ii) The weak purchasing power of COPD Tunisian patients (i.e., inability to access the treatments); (iii) patients living in rural areas (25.7\% in this study) had more difficulties to obtain their treatment; and (iv) different national and cultural attitudes of Tunisian pulmonologists to various classes of drugs [50].

4.2.2. Adherence Barriers to the 2017-GOLD PTGs. In COPD, some specific variables, largely described by López-Campos et al. [12], are associated with PTG adherence. Some of them are physician- (e.g., knowledge), patient- (e.g., understanding), and/or social- (e.g., costs of medication) related factors [17-23]. The Tunisian pulmonologists' adherence to the 2017-GOLD PTGs is influenced by the patients' age, SEL, national health insurance coverage, and GOLD groups (Table 5). Solving the problems related to each of the aforementioned factors will improve the adherence to PTGs [14]. The following sentences will discuss the aforementioned four factors.

The adherence rate increased in older patients (Table 5). This result is in contrast with the one reported by Sharif et al. [49]. They showed that the 2007 COPD PTGs concordant and discordant groups have similar ages [49]. One explanation for this study result could be the coexistence, in elderly patients, of some frequent comorbidities, especially arterial hypertension and diabetes mellitus (Table 2). In fact, most COPD male patients with concurrent cardiovascular disease are more likely to be prescribed bronchodilators [51]. However, since medical treatments of diabetes mellitus and arterial hypertension are totally funded by the national health insurance, Tunisian pulmonologists are sure that COPD treatments will also be reimbursed.

The patient's socioeconomic factors and low-income status have been shown to influence the adherence rate and to be related to the nonuse of medication $[14,52]$. Tottenborg et al. [52] examined the impact of both employment and income on the risk of suboptimal adherence to inhaled medication among COPD patients. They showed a higher risk of poor adherence among unemployed (adjusted related risks (aRR): 1.36) and low-income patients (aRR: 1.07). Barriers to adherence related to low income include inconsistent primary healthcare, inability to pay for COPD treatments, and lack of transport [14].

The adherence rate was 2.851 times higher in patients benefiting of the national health insurance coverage when compared to those indigent or without insurance (Table 5). In case of the nonavailability of a national insurance coverage, pulmonologists avoid to prescribe some inhaled drugs (especially LABA and LAMA) since their prices are high and they are sure that patients will not be able to purchase them. The lack of access to resources is also an important barrier. It contributes not only to inappropriate medical treatments but also to under referral of patients to COPD educators and pulmonary rehabilitation [12]. Moreover, it seems that the insurance coverage rules for medications may influence the physicians' prescribing patterns by deviating from the evidence-based guidelines [53].

The adherence rate was almost three times higher in patients classified A/B GOLD groups when compared to those classified C/D (Table 5). This finding is partially in line with those reported in some related studies $[6,9,13]$ (Appendix A). First, after adjusting for age, sex, smoking habits, number of exacerbations, control of symptoms, and health service use, Maio et al. [6] showed that group D patients, compared to the ones in group A, are significant protective factors for the lack of prescriptive appropriateness (OR: $0.05)$. Second, it seems that $8.9 \%$ of group D patients are prescribed single bronchodilator therapy by pulmonologists [16]. Pulmonologists also prescribed ICS-deterio triple therapy to $14.3,27.1$, and $57.1 \%$ of patients belonging to groups A, B, and C, respectively [16]. Third, Ding et al. [16] reported that patients in groups $\mathrm{A}$ and $\mathrm{D}$ are more likely to be treated in line with the 2014-GOLD PTGs (61.5 and 77.5\%, respectively), compared with $40.1 \%$ for group B. Fourth, Palmiotti et al. [13] showed that 49 and $46 \%$ of patients in groups A and B were following therapies differently from the 2015GOLD PTGs, that $41.5 \%$ of patients in group C received triple combination therapy, and that $14 \%$ of patients in group D did not have a therapy or were following an inappropriate therapy.

In an Italian study, pulmonologists were asked to respond to an online survey aiming to identify the barriers to the 2015-GOLD PTGs and therefore to explain the discrepancy between the guidelines and the clinicians' practices [13]. It appears that (i) $30 \%$ of the discrepancy is due to the fact that GOLD recommendations are far from reality; (ii) the "ABCD model" is difficult to regularly use since spirometry is not always possible and it increases the visit duration; (iii) ICS overprescription is due to the information received from the pharmaceutical companies and because ICS are more effective than proven in clinical trials; (iv) inappropriate use of triple therapy is due to the belief that it guarantees better and faster results with regard to the patient's symptoms and it is easier to prescribe than double bronchodilation alone; and (v) physicians and patients underestimate the disease causing $11 \%$ of group $\mathrm{D}$ patients not to be treated. The following three additional barriers, described in Appendix A, were advanced by pulmonologists: disagreement with the guidelines [48], influence of the clinicians' judgment with regard to the validity of some guidelines [49], and lack of perceived benefits [19].

To conclude, the main result of this study was that the adherence rate of North-African pulmonologists to the 2017-COPD PTGs was disappointingly low. Once again, the study results support the established discrepancy between the current real-life practice and the GOLD PTGs in terms of the low reference to COPD management guidelines by pulmonologists, proving that adherence to the GOLD PTGs strategy is far from being optimal.

\section{Abbreviations}

aRR: $\quad$ Adjusted relative risks

CAT: COPD assessment test

COPD: Chronic obstructive pulmonary disease

$\mathrm{FEV}_{1}$ : Forced expiratory volume in one second

FVC: $\quad$ Forced vital capacity

GOLD: Global Initiative for Chronic Obstructive Lung Disease 
ICS: Inhaled corticosteroids

LABA: Long-acting $\beta$-agonist

LAMA: Long-acting muscarinic antagonist

mMRC: Modified British Medical Research Council

OR: Odds ratio

PBD: Postbronchodilator

PTGs: Pharmacological treatment guidelines

SABA: Short-acting $\beta$-agonist

SAMA: Short-acting muscarinic antagonist

SEL: $\quad$ Socioeconomic level

WHO: World Health Organization.

\section{Data Availability}

The data used to support the findings of this study are available from the corresponding author upon request.

\section{Conflicts of Interest}

HBS reports personal fees from AstraZeneca, Saiph, Teriak, Hikma, Chiesi, and Opalia Recordati. The remaining authors declare that they have no conflicts of interest concerning this article.

\section{Authors' Contributions}

SA, AsK, JM, AhK, and HBS are responsible for literature search, data collection, study design, analysis of data, manuscript preparation, and review of manuscript. WB, AA, AG, $\mathrm{AH}, \mathrm{MB}$, and IG are responsible for manuscript preparation and review of manuscript. All authors read and approved the final manuscript. Sana AISSA and Asma KNAZ contributed equally as first authors to this study.

\section{Acknowledgments}

Authors wish to thank Professor Samir BOUKATTAYA for his invaluable contribution in the improvement of the quality of the writing in the present paper. Department of Pneumology, Farhat Hached University Hospital in Sousse, Tunisia, is the name and location of the institution where the study was performed.

\section{Supplementary Materials}

The appendix includes the following sections: population and methods (i.e., sample size calculation, dyspnea evaluation, and description of the 4 GOLD stages), results (i.e., population general characteristics), and discussion (i.e., rational of the study, discussion of methodology, and description of the adherence barriers to the 2017-GOLD PTGs). Moreover, it includes a table summarizing the adherence variability to some GOLD PTGs observed in the literature. (Supplementary Materials)

\section{References}

[1] C. F. Vogelmeier, G. J. Criner, F. J. Martinez et al., "Global strategy for the diagnosis, management, and prevention of chronic obstructive lung disease 2017 report. GOLD executive summary," American Journal of Respiratory and Critical Care Medicine, vol. 195, no. 5, pp. 557-582, 2017.

[2] M. W. Hess, "The 2017 Global Initiative for Chronic Obstructive Lung Disease report and practice implications for the respiratory therapist," Respiratory Care, vol. 62, no. 11, pp. 1492-1500, 2017.

[3] The Lancet, "Complexities of care in COPD," The Lancet, vol. 389, no. 10069, p. 574, 2017.

[4] B. Alcazar-Navarrete, F. Castellano Minan, and P. J. Romero Palacios, "Clinical guidelines in asthma and chronic obstructive pulmonary disease: how useful are they in clinical practice?," Archivos de Bronconeumología, vol. 54, no. 3, pp. 117-118, 2018.

[5] E. Stafyla, O. S. Kotsiou, K. Deskata, and K. I. Gourgoulianis, "Missed diagnosis and overtreatment of COPD among smoking primary care population in Central Greece: old problems persist," International Journal of Chronic Obstructive Pulmonary Disease, vol. Volume 13, pp. 487-498, 2018.

[6] S. Maio, S. Baldacci, F. Martini et al., "COPD management according to old and new GOLD guidelines: an observational study with Italian general practitioners," Current Medical Research and Opinion, vol. 30, no. 6, pp. 1033-1042, 2014.

[7] H. D. Foda, A. Brehm, K. Goldsteen, and N. H. Edelman, "Inverse relationship between nonadherence to original GOLD treatment guidelines and exacerbations of COPD," International Journal of Chronic Obstructive Pulmonary Disease, vol. Volume 12, pp. 209-214, 2017.

[8] P. C. Chavez and N. K. Shokar, "Diagnosis and management of chronic obstructive pulmonary disease (COPD) in a primary care clinic," COPD, vol. 6, no. 6, pp. 446-451, 2009.

[9] M. J. Hsieh, S. Y. Huang, T. M. Yang et al., "The impact of 2011 and 2017 global initiative for chronic obstructive pulmonary disease (GOLD) guidelines on allocation and pharmacological management of patients with COPD in Taiwan: Taiwan obstructive lung disease (TOLD) study," International Journal of Chronic Obstructive Pulmonary Disease, vol. 13, pp. 29492959, 2018.

[10] T.-O. Kim, H.-J. Shin, Y.-I. Kim et al., "Adherence to the GOLD guideline in COPD management of South Korea: findings from KOCOSS study 2011-2018," Chonnam Medical Journal, vol. 55, no. 1, pp. 47-53, 2019.

[11] C. R. Pinto, A. C. M. Lemos, L. Assunção-Costa et al., "Management of COPD within the Brazilian unified health care system in the state of Bahia: an analysis of real-life medication use patterns," Jornal Brasileiro de Pneumologia, vol. 45, no. 1, 2019.

[12] J. L. López-Campos, E. Q. Gallego, and L. C. Hernández, "Status of and strategies for improving adherence to COPD treatment," International Journal of Chronic Obstructive Pulmonary Disease, vol. Volume 14, pp. 1503-1515, 2019.

[13] G. A. Palmiotti, D. Lacedonia, V. Liotino et al., "Adherence to GOLD guidelines in real-life COPD management in the Puglia region of Italy," International Journal of Chronic Obstructive Pulmonary Disease, vol. Volume 13, pp. 24552462, 2018.

[14] Adherence to long-term therapies: evidence for action, World Health Organization, Geneva, Switzerland, 2003, February 2019, https://www.who.int/chp/knowledge/publications/ adherence_report/en/.

[15] A. Corrado and A. Rossi, "How far is real life from COPD therapy guidelines? An Italian observational study," Respiratory Medicine, vol. 106, no. 7, pp. 989-997, 2012. 
[16] B. Ding, M. Small, and U. Holmgren, "A cross-sectional survey of current treatment and symptom burden of patients with COPD consulting for routine care according to GOLD 2014 classifications," International Journal of Chronic Obstructive Pulmonary Disease, vol. 12, pp. 1527-1537, 2017.

[17] M. D. Cabana, C. S. Rand, N. R. Powe et al., "Why don't physicians follow clinical practice guidelines? A framework for improvement," JAMA, vol. 282, no. 15, pp. 1458-1465, 1999.

[18] O. O. Desalu, C. C. Onyedum, A. O. Adeoti et al., "Guideline-based COPD management in a resource-limited setting - physicians' understanding, adherence and barriers: a cross-sectional survey of internal and family medicine hospital-based physicians in Nigeria," Primary Care Respiratory Journal, vol. 22, no. 1, pp. 79-85, 2013.

[19] X. Perez, J. P. Wisnivesky, L. Lurslurchachai, L. C. Kleinman, and I. M. Kronish, "Barriers to adherence to COPD guidelines among primary care providers," Respiratory Medicine, vol. 106, no. 3, pp. 374-381, 2012.

[20] C. C. López, C. C. Macario, Y. M. Blazquez et al., “Agreement between a simple dyspnea-guided treatment algorithm for stable COPD and the GOLD guidelines: a pilot study," International Journal of Chronic Obstructive Pulmonary Disease, vol. 11, pp. 1217-1222, 2016.

[21] H. Sandelowsky, I. Hylander, I. Krakau, S. Modin, B. Stallberg, and A. Nager, "Time pressured deprioritization of COPD in primary care: a qualitative study," Scandinavian Journal of Primary Health Care, vol. 34, no. 1, pp. 55-65, 2016.

[22] S. P. E. Nishi, M. Maslonka, W. Zhang, Y. F. Kuo, and G. Sharma, "Pattern and adherence to maintenance medication use in medicare beneficiaries with chronic obstructive pulmonary disease: 2008-2013," Chronic Obstructive Pulmonary Diseases, vol. 5, no. 1, pp. 16-26, 2018.

[23] M. E. Alsubaiei, P. A. Frith, P. A. Cafarella et al., "COPD care in Saudi Arabia: physicians' awareness and knowledge of guidelines and barriers to implementation," The International Journal of Tuberculosis and Lung Disease, vol. 21, no. 5, pp. 592-595, 2017.

[24] H. Daldoul, M. Denguezli, A. Jithoo et al., "Prevalence of COPD and tobacco smoking in Tunisia-results from the BOLD study," International Journal of Environmental Research and Public Health, vol. 10, no. 12, pp. 7257-7271, 2013.

[25] M. Kang, B. G. Ragan, and J. H. Park, "Issues in outcomes research: an overview of randomization techniques for clinical trials," Journal of Athletic Training, vol. 43, no. 2, pp. 215-221, 2008.

[26] D. Pevalin and D. Rose, "The national statistics socioeconomic classification : unifying official and sociological approaches to the conceptualisation and measurement of social class in the united kingdom," Sociétés contemporaines, vol. 45-46, no. 1, pp. 75-106, 2002.

[27] J. Reichert, A. J. de Araújo, C. M. C. Gonçalves et al., "Smoking cessation guidelines-2008," Jornal Brasileiro de Pneumologia, vol. 34, no. 10, pp. 845-880, 2008.

[28] J. L. López-Campos, A. Fernández-Villar, C. Calero-Acuña et al., "Occupational and biomass exposure in chronic obstructive pulmonary disease: results of a cross-sectional analysis of the on-sint study," Archivos de Bronconeumología, vol. 53, no. 1, pp. 7-12, 2017.

[29] C. M. Fletcher, P. C. Elmes, A. S. Fairbairn, and C. H. Wood, "The significance of respiratory symptoms and the diagnosis of chronic bronchitis in a working population," British Medical Journal, vol. 2, no. 5147, pp. 257-266, 1959.

[30] M. R. Miller, R. Crapo, J. Hankinson et al., "General considerations for lung function testing," The European Respiratory Journal, vol. 26, no. 1, pp. 153-161, 2005.

[31] H. Ben Saad, M. N. El Attar, K. H. Mabrouk et al., "The recent multi-ethnic global lung initiative $2012\left(\mathrm{GLI}_{2012}\right)$ reference values don't reflect contemporary adult's North African spirometry," Respiratory Medicine, vol. 107, no. 12, pp. 20002008, 2013.

[32] A. R. Anzueto, K. Kostikas, K. Mezzi et al., "Indacaterol/glycopyrronium versus salmeterol/fluticasone in the prevention of clinically important deterioration in COPD: results from the FLAME study," Respiratory Research, vol. 19, no. 1, p. 121, 2018.

[33] J. R. Hurst, J. Vestbo, A. Anzueto et al., "Susceptibility to exacerbation in chronic obstructive pulmonary disease," The New England Journal of Medicine, vol. 363, no. 12, pp. 1128-1138, 2010.

[34] PubMed, February 24, 2020 https://www.ncbi.nlm.nih.gov/ $\mathrm{mesh} /$ ?term $=$ adherence.

[35] L. M. Hess, M. A. Raebel, D. A. Conner, and D. C. Malone, "Measurement of adherence in pharmacy administrative databases: a proposal for standard definitions and preferred measures," The Annals of Pharmacotherapy, vol. 40, no. 7-8, pp. 1280-1288, 2006.

[36] E. Van Ganse and D. Price, "Respiratory medication adherence: toward a common language and a shared vision," The Journal of Allergy and Clinical Immunology. In Practice, vol. 4, no. 5, pp. 799-801, 2016.

[37] M. Park, "Using patient medical records for medical research," Korean Journal of Family Medicine, vol. 34, no. 3, p. 159, 2013.

[38] A. L. Terry, V. Chevendra, A. Thind, M. Stewart, J. N. Marshall, and S. Cejic, "Using your electronic medical record for research: a primer for avoiding pitfalls," Family Practice, vol. 27, no. 1, pp. 121-126, 2010.

[39] A. M. Euser, C. Zoccali, K. J. Jager, and F. W. Dekker, "Cohort studies: prospective versus retrospective," Nephron. Clinical Practice, vol. 113, no. 3, pp. c214-c217, 2009.

[40] J. Sehl, J. O’Doherty, R. O'Connor, B. O'Sullivan, and A. O'Regan, “Adherence to COPD management guidelines in general practice? A review of the literature," Irish Journal of Medical Science, vol. 187, no. 2, pp. 403-407, 2018.

[41] M. Papala, N. Kerenidi, and K. I. Gourgoulianis, "Everyday clinical practice and its relationship to 2010 and 2011 GOLD guideline recommendations for the management of COPD," Primary Care Respiratory Journal, vol. 22, no. 3, pp. 362-364, 2013.

[42] E. Bertella, A. Zadra, M. Vitacca, and on behalf of General Practitioners Distretto 04 ASL 02 Brescia, Italy, "COPD management in primary care: is an educational plan for GPs useful?," Multidisciplinary Respiratory Medicine, vol. 8, no. 1, p. 24, 2013.

[43] K. K. P. Chan, F. Ko, H. S. Chan et al., "Adherence to a COPD treatment guideline among patients in Hong Kong," International Journal of Chronic Obstructive Pulmonary Disease, vol. Volume 12, pp. 3371-3379, 2017.

[44] E. Sen, S. Z. Guclu, I. Kibar et al., "Adherence to GOLD guideline treatment recommendations among pulmonologists in\&nbsp;Turkey," International Journal of Chronic Obstructive Pulmonary Disease, vol. 10, pp. 2657-2663, 2015. 
[45] D. Spyratos, D. Chloros, D. Michalopoulou, and L. Sichletidis, "Estimating the extent and economic impact of under and overdiagnosis of chronic obstructive pulmonary disease in primary care," Chronic Respiratory Disease, vol. 13, no. 3, pp. 240-246, 2016.

[46] Y. Ivanov, I. Nikolaev, and I. Nemeth, "Real-life evaluation of COPD treatment in a Bulgarian population: a 1-year prospective, observational, noninterventional study," International Journal of Chronic Obstructive Pulmonary Disease, vol. 13, pp. 653-663, 2018.

[47] Y. F. Wei, P. H. Kuo, Y. H. Tsai et al., "Factors associated with the prescription of inhaled corticosteroids in GOLD group A and $\mathrm{B}$ patients with COPD - subgroup analysis of the Taiwan obstructive lung disease cohort," International Journal of Chronic Obstructive Pulmonary Disease, vol. 10, pp. 19511956, 2015.

[48] K. J. Davis, S. H. Landis, Y. M. Oh et al., "Continuing to confront COPD international physician survey: physician knowledge and application of COPD management guidelines in 12 countries," International Journal of Chronic Obstructive Pulmonary Disease, vol. 10, pp. 39-55, 2015.

[49] R. Sharif, C. R. Cuevas, Y. Wang, M. Arora, and G. Sharma, "Guideline adherence in management of stable chronic obstructive pulmonary disease," Respiratory Medicine, vol. 107, no. 7, pp. 1046-1052, 2013.

[50] M. Rudolf, "The reality of drug use in COPD: the European perspective," Chest, vol. 117, no. 2, Supplement, pp. 29S-32S, 2000.

[51] D. T. Adesanoye and C. J. Willey, "Does cardiovascular comorbidity influence the prescribing of bronchodilators in chronic obstructive pulmonary disease?," The Annals of Pharmacotherapy, vol. 51, no. 10, pp. 855-861, 2017.

[52] S. S. Tottenborg, P. Lange, S. P. Johnsen, H. Nielsen, T. S. Ingebrigtsen, and R. W. Thomsen, "Socioeconomic inequalities in adherence to inhaled maintenance medications and clinical prognosis of COPD," Respiratory Medicine, vol. 119, pp. 160-167, 2016.

[53] P. Hernandez, M. S. Balter, J. Bourbeau, C. K. Chan, D. D. Marciniuk, and S. L. Walker, "Canadian practice assessment in chronic obstructive pulmonary disease: respiratory specialist physician perception versus patient reality," Canadian Respiratory Journal, vol. 20, no. 2, pp. 97-105, 2013. 